\title{
CONVEX MEROMORPHIC MAPPINGS AND RELATED FUNCTIONS
}

\section{JAMES MILLER}

1. Introduction. Let $U(p)$ denote the class of univalent meromorphic functions $f(z)$ in the unit disk $E$ with a simple pole at $z=p>0$ and with the normalization $f(0)=0$ and $f^{\prime}(0)=1$. Let $K(p)$ denote the functions which belong to $U(p)$ and map $|z|\langle r\rangle \rho$ (for some $p<\rho<1)$ onto the complement of a convex set. If $f(z) \in K(p)$, then there is a $\rho, p<\rho<1$, such that for each $z, \rho<|z|<1$

$$
\operatorname{Re}\left\{1+z f^{\prime \prime}(z) / f^{\prime}(z)\right\} \leqq 0 .
$$

If $f \in K(p)$, then for each $z$ in $E$,

$$
\operatorname{Re}\left\{1+z \frac{f^{\prime \prime}(z)}{f^{\prime}(z)}+\frac{2 p}{z-p}-\frac{2 p z}{1-p z}\right\} \leqq 0 .
$$

Let $\Sigma(p)$ denote the class of mappings $f(z)$ which satisfy (1) and the conditions $f(0)=0$ and $f^{\prime}(0)=1$. The class $K(p)$ is contained in $\Sigma(p)$. Royster [9] has shown that for $0<p<2-\sqrt{ } 3$, if $f(z) \in \Sigma(p)$ and is meromorphic, then $f(z) \in K(p)$. The class $U(p)$ and related classes have been studied in [1], [2], [4], and [5].

In this paper, we shall be interested in examining functions in $K(p)$ and $\Sigma(p)$ with respect to their properties near the origin. In particular, we shall examine the radius of convexity of $K(p)$ and $\Sigma(p)$. We shall also define and study close-to-convex functions and functions which are starlike with respect to some point in the complement of $f(0)$.

2. Convex functions. Let $P$ denote the class of functions $P(z)$ which are regular in $E$ and satisfy $P(0)=1$ and $\operatorname{Re}\{P(z)\} \geqq 0$ for all $z \in E$. For each function $f(z) \in \Sigma(p)$, there is a function $P(z) \in P$ such that

$$
1+z \frac{f^{\prime \prime}(z)}{f^{\prime}(z)}+\frac{2 p}{z-p}-\frac{2 p z}{1-p z}=-P(z) .
$$

Taking real parts, we have

$$
\operatorname{Re}\left\{1+z \frac{f^{\prime \prime}(z)}{f^{\prime}(z)}\right\}=2 p \operatorname{Re}\left\{\frac{z}{1-p z}-\frac{1}{z-p}\right\}-\operatorname{Re}\{P(z)\} .
$$

Presented to the Society, April 18, 1969; received by the editors March 10, 1969. 
To ${ }^{\prime}$ find the radius of convexity for $\Sigma(p)$, we need the following lemma:

Lemma 1. Let

$$
Q(r, \theta)=\operatorname{Re}\left\{\frac{z}{1-p z}-\frac{1}{z-p}\right\}
$$

where $z=r e^{i \theta}$ and $0<p<1$. Then for $r<p$, we have

$$
\min _{0 \leqq \theta<2 \pi} Q(r, \theta)=Q(r, \pi)=\frac{1-r^{2}}{(p+r)(1+p r)} .
$$

Proof. Computing $Q(r, \theta)$ yields

$$
Q(r, \theta)=\frac{r \cos \theta-p r^{2}}{1-2 p r \cos \theta+p^{2} r^{2}}+\frac{p-r \cos \theta}{r^{2}-2 p r \cos \theta+p^{2}} .
$$

For a fixed $r$, we have

$$
\frac{d Q}{d \theta}=\frac{-r q(r, \theta) \sin \theta}{\left(1-2 p r \cos \theta+p^{2} r^{2}\right)^{2}\left(r^{2}-2 p r \cos \theta+p^{2}\right)^{2}}
$$

where

$q(r, \theta)=\left(1-p^{2} r^{2}\right)\left(r^{2}-2 p r \cos \theta+p^{2}\right)^{2}+\left(p^{2}-r^{2}\right)\left(1-2 p r \cos \theta+p^{2} r^{2}\right)^{2}$. If $^{3} r<p$, we see that $q(r, \theta)>0$ for all $\theta, 0 \leqq \theta<2 \pi$. Thus for $r<p, Q(r, \theta)$ will have its minimum at $\theta=\pi$. This proves the lemma.

THEOREM 1. If $f(z) \in \Sigma(p)$, then $f(z)$ maps $|z|<\rho_{0}(p)$ onto a convex set, where

$$
\rho_{0}(p)=\left[1+4 p+p^{2}-(p+1)\left(p^{2}+6 p+1\right)^{1 / 2}\right] / 2 p .
$$

Proof. For $P(z) \in \mathcal{P}$, it is well known that

$$
\max _{P \in \odot} \max _{|z|=r} \operatorname{Re}\{P(z)\}=\frac{1+r}{1-r} .
$$

If $f(z)$ satisfies the hypotheses of the theorem, then (3), (4), and $(5)$ give

$$
\begin{aligned}
\operatorname{Re}\left\{1+z \frac{f^{\prime \prime}(z)}{f^{\prime}(z)}\right\} & \geqq \frac{2 p\left(1-r^{2}\right)}{(p+r)(1+p r)}-\frac{1+r}{1-r} \\
& =\frac{p-\left(1+3 p+p^{2}\right)\left(r+r^{2}\right)+p r^{3}}{(p+r)(1+p r)(1-r)}
\end{aligned}
$$

for $|z|<p$. This proves the theorem. 
A brief calculation shows that a function $f(z) \in \Sigma(p)$ is meromorphic in $E$ if and only if the function $P(z)$ given in (2) has the properties

(i) $P(z) \in P$ and

(ii) $P(p)=\left(1+p^{2}\right)\left(1-p^{2}\right)^{-1}$.

Thus for a function $f(z) \in K(p)$, equation (2) holds where the function $P(z)$ satisfies (i) and (ii).

To find the radius of convexity for $K(p)$, first we must prove the following lemma.

Lemma 2. Let $P(z) \in \rho$ and $P(p)=\left(1+p^{2}\right)\left(1-p^{2}\right)^{-1}, 0<p<1$. Then for $r<p / 2$,

$$
\max _{|z|=r} \operatorname{Re}\{P(z)\} \leqq \frac{\left(1+p^{2}\right)\left(1-r^{2}\right)}{(1-r p)^{2}+(r-p)^{2}} .
$$

Proof. Let $w(z)=(P(z)-1)(P(z)+1)^{-1}$. Then $w(z)$ is regular in $E$ and satisfies $w(0)=0, w(p)=p^{2}$, and $|w(z)|<1$ for $z \in E$. Define the following functions:

$$
g(z)=\frac{w(z)-p^{2}}{1-p^{2} w(z)} \cdot \frac{1-p z}{z-p}, \quad h(z)=-\frac{g(z)-p}{1-p g(z)} .
$$

Then $h(z)$ is regular in $E, h(0)=0$, and $|h(z)|<1$. Thus $|h(z)| \leqq z$. Let $\zeta=(z-p)(1-p z)^{-1}$, then we may write

$$
P(z)=\frac{1+p^{2}}{1-p^{2}} \cdot \frac{1+g(z) \zeta}{1-g(z) \zeta} .
$$

Now, the problem max $\operatorname{Re}\{P(z)\}$ is equivalent to

$$
\max _{|z|=r} \operatorname{Re}\left\{\frac{1+g(z) \zeta}{1-g(z) \zeta}\right\}=\max _{|z|=r} \frac{1-|g(z) \zeta|^{2}}{|1-g(z) \zeta|^{2}} .
$$

Let $z$ satisfy $|z|=r$; then $\zeta=(z-p)(1-p z)^{-1}$ lies on the circle $C_{1}$ : $|\zeta+a|=R$ where $a=p\left(1-r^{2}\right)\left(1-p^{2} r^{2}\right)^{-1}$ and $R=r\left(1-p^{2}\right)\left(1-r^{2} p^{2}\right)^{-1}$. Since $|h(z)| \leqq|z|$, the point $g(z)$ lies in the closed disk $C_{2}:|g(z)-a|$ $\leqq R$. The quantity $|g(z) \zeta|$ is minimum when $g(z)=a-R$ and $\zeta=-a+R$. We will show that $\max \operatorname{Re}\{P(z)\}$ occurs at the same point as $\min |g(z) \zeta|$ provided $R<a / 2$. Let $\zeta_{0}=-a+R e^{i \theta}$ lie on $C_{1}$. Then $g(z) \zeta_{0}$ lies in the circle $C_{3}:\left|g(z) \zeta_{0}-\zeta_{0} a\right| \leqq\left|\zeta_{0}\right| R$. The distance from 1 to the circle is $d(\theta)=\left|1-\zeta_{0} a\right|-\left|\zeta_{0}\right| R$. We observe that $\min _{0 \leqq \theta<2 \pi} d(\theta)=\min _{|z|-r}|1-g(z) \zeta|$. The function $d(\theta)$ is a minimum at $\theta=0$ provided $\left|a^{2} R^{2}-a^{2}\left(1+a^{2}\right)\right|>2\left|\left(1+a^{2}\right) a R^{3}-a R\right|$ which holds for $R<a / 2$. Thus for $r<p / 2$ we have shown 


$$
\frac{1-|g(z) \zeta|^{2}}{|1-g(z) \zeta|^{2}} \leqq \frac{1-(a-R)^{2}}{1+(a-R)^{2}}=\frac{\left(1-p^{2}\right)\left(1-r^{2}\right)}{(1-p r)^{2}+(p-r)^{2}}
$$

which proves the lemma. Equality occurs when $h(z)=z$ at $z=r$.

Applying Lemmas 1 and 2 to equation (3), we have the following:

Theorem 2. If $f(z) \in K(p)$, then

$$
\operatorname{Re}\left\{1+z\left(f^{\prime \prime}(z) / f^{\prime}(z)\right)\right\}>0
$$

for $|z|<\min \left[p / 2, \rho_{1}(p)\right]$ where $\rho_{1}(p)$ is the smallest positive root of

$$
p^{3}+p-\left(1+10 p^{2}+p^{4}\right) r+\left(p^{3}+p\right) r^{2}=0 .
$$

For $.1<p<1$, we have $\rho_{1}(p)<p / 2$.

The number $\rho_{0}(p)$ for $\Sigma(p)$ in Theorem 1 is best possible, whereas the number $\rho_{1}(p)$ in Theorem 2 is not. As $p$ tends to 1 , both $\rho_{0}(p)$ and $\rho_{1}(p)$ tend to $3-2 \sqrt{ } 2$.

3. Close-to-convex functions. Let us define a class of close-toconvex functions. A function $f(z)\left(f(0)=0, f^{\prime}(0)=1\right)$ belongs to the class $C \Sigma(p)$ (close-to-convex functions) if $f(z)$ is regular at each point of $E$ except at $p$ and if there is a function $e^{i \Phi} g(z) \in \Sigma(p),|\Phi| \leqq \pi / 2$, such that

$$
\operatorname{Re}\left\{f^{\prime}(z) / g^{\prime}(z)\right\}>0
$$

for all $z \in E$. Let $C \Sigma_{0}(p)$ denote the subclass of $C \Sigma(p)$ for which $\Phi=0$. A function in $C \Sigma(p)$ may have a logarithmic singularity at $z=p$ even though the corresponding function $g(z)$ is meromorphic in $E$.

Let $f(z) \in C \Sigma(p)$ and $e^{i \Phi} g(z) \in \Sigma(p)$ be functions such that

$$
\operatorname{Re}\left\{f^{\prime}(z) / g^{\prime}(z)\right\} \geqq 0
$$

for all $z \in E$. Define the functions $P_{1}(z)$ and $Q_{1}(z)$ such that $i \exp \left[i P_{1}(z)\right]$ and $i \exp \left[i Q_{1}(z)\right]$ are the unit tangent vectors to the images of $|z|=r$ under $f(z)$ and $g(z)$, respectively. Since (7) holds, we have (provided the proper choice of arguments has been made)

$$
\left|P_{1}(z)-Q_{1}(z)\right|<\pi / 2 .
$$

Define $Q(r, \theta)=Q_{1}(z)+\arg \left[(z-p)^{2}(1-p z)^{2} z^{-2}\right]$ and $P(r, \theta)=P_{1}(z)$ $+\arg \left[(z-p)^{2}(1-p z)^{2} z^{-2}\right]$ where $z=r e^{i \theta}$. Since $e^{i \Phi} g(z) \in \Sigma(p)$, we have $\partial Q / \partial \theta<0$. An argument similar to one used by Kaplan [3] yields

$$
P\left(r, \theta_{1}\right)-P\left(r, \theta_{2}\right)>-\pi \text { for } \theta_{1}<\theta_{2},
$$


which is equivalent to

$$
\int_{\theta_{1}}^{\theta_{2}} \operatorname{Re}\left\{1+r e^{i \theta} \frac{f^{\prime \prime}\left(r e^{i \theta}\right)}{f^{\prime}\left(r e^{i \theta}\right)}\right\} d \theta
$$

$$
\begin{array}{r}
<\pi-\arg \left[\left(r e^{i \theta_{2}}-p\right)^{2}\left(1-p r e^{i \theta_{2}}\right)^{2} r^{-2} e^{-2 i \theta_{2}}\right] \\
+\arg \left[\left(r e^{i \theta_{1}}-p\right)^{2}\left(1-p r e^{i \theta_{1}}\right)^{2} r^{-2} e^{-2 i \theta_{1}}\right] .
\end{array}
$$

Thus (7) implies (8).

To prove the converse, we define $P(r, \theta)$ as defined above and assume that (8) holds. Since $f(z)$ may have a logarithmic singularity, we cannot use Libera and Robertson's proof, although the proof given here is similar to both their proof and Kaplan's. From the definition of $P(r, \theta)$, we see $P(r, \theta+2 \pi)-P(r, \theta)=-2 \pi$. We now use

Lemma 3 [6]. Let $t(\theta)$ be a real function of $\theta$ for $-\infty<\theta<\infty$ such that

$$
\begin{gathered}
t(\theta+2 \pi)-t(\theta)=-2 \pi, \\
t\left(\theta_{1}\right)-t\left(\theta_{2}\right)>-\pi \text { for } \theta_{1}>\theta_{2} .
\end{gathered}
$$

Then there exists a real valued function $s(\theta)$ which is nonincreasing and satisfies the conditions

$$
\begin{gathered}
s(\theta+2 \pi)-s(\theta)=-2 \pi, \\
|s(\theta)-t(\theta)| \leqq \pi / 2 .
\end{gathered}
$$

For a fixed $\rho$, let $t(\theta)=P(\rho, \theta)$ and denote a corresponding $s(\theta)$ which is given in Lemma 3 by $s(\rho, \theta)$. For $r<\rho$, define $q_{\rho}(r, \theta)$ by

$$
q_{\rho}(r, \theta)=\frac{\rho^{2}-r^{2}}{2 \pi} \int_{0}^{2 \pi} \frac{s(\rho, \alpha)+\alpha}{\rho^{2}-2 \rho r \cos (\theta-\alpha)+r^{2}} d \alpha .
$$

The function $q_{\rho}(r, \theta)$ is harmonic for $r<\rho$. Now define

$$
Q_{\rho}(r, \theta)=q_{\rho}(r, \theta)-\theta .
$$

Since $s\left(\rho, \alpha+\theta_{2}\right)-s\left(\rho, \alpha+\theta_{1}\right)$ is nonpositive for $\theta_{1}<\theta_{2}$ and has period $2 \pi$, we may write

$$
Q_{\rho}\left(r, \theta_{2}\right)-Q_{\rho}\left(r, \theta_{1}\right)=\frac{\rho^{2}-r^{2}}{2 \pi} \int_{0}^{2 \pi} \frac{s\left(\rho, \alpha+\theta_{2}\right)-s\left(\rho, \alpha+\theta_{1}\right)}{\rho^{2}-2 \rho r \cos \alpha+r^{2}} d \alpha<0 .
$$

Let $h_{\rho}(z)$ be the analytic completion of $q_{\rho}(r, \theta)$ such that $\operatorname{Im} h_{\rho}(z)$ $=q_{\rho}(r, \theta)$ and $\operatorname{Re} h_{\rho}(0)=0$. Define $g_{\rho}(z)$ to be 


$$
g_{\rho}(z)=p^{2} \int_{0}^{z} \frac{e^{h_{\rho}(z)}}{(z-p)^{2}(1-p z)^{2}} d z .
$$

If $|z|<\rho$, then $g_{\rho}(z)$ satisfies

$$
\begin{aligned}
\operatorname{lo} g_{\rho}^{\prime}(0) \mid=1, & \\
\operatorname{Re}\left\{1+z \frac{g^{\prime \prime}(z)}{g^{\prime}(z)}+\frac{2 p}{z-p}-\frac{2 p z}{1-p z}\right\}= & \operatorname{Re}\left\{z h_{\rho}^{\prime}(z)-1\right\} \\
& =\frac{\partial Q_{\rho}(r, \theta)}{\partial \theta}<0, \\
\operatorname{Re}\left\{f^{\prime}(z) / g_{\nu}^{\prime}(z)\right\}>0 . &
\end{aligned}
$$

The function $F_{\rho}(z)=e^{h_{\rho}^{(z)}} / z$ is a univalent starlike function in $0<|z|<\rho$. Following the argument of Kaplan [3] we can choose a sequence $\rho_{n} \rightarrow 1$ such that $F_{\rho_{n}}$ converges uniformly to $F(z)$ in every closed domain of $E$. The function $F(z)$ is univalent and starlike in $0<|z|<1$, and the function

$$
g^{\prime}(z)=p^{2} \frac{z F(z)}{(z-p)^{2}(1-p z)^{2}}
$$

satisfies (1) and

$$
\operatorname{Re}\left\{f^{\prime}(z) / g^{\prime}(z)\right\}>0
$$

for all $|z|<1$. Thus (8) is an equivalent condition for a function to belong to $C \Sigma(\rho)$.

Remark. Suppose $p=0$; then condition (1) for $g(z)$ is

$$
\operatorname{Re}\left\{1+z g^{\prime \prime}(z) / g^{\prime}(z)\right\}<0 .
$$

Thus $F(z)=z g^{\prime}(z)$ is starlike with respect to the origin in $0<|z|<1$. Equations (7) and (8) become

$$
\operatorname{Re}\left\{z f^{\prime}(z) / f(z)\right\}>0
$$

and

$$
\int_{\theta 1}^{\theta_{2}} \operatorname{Re}\left\{1+r e^{i \theta} \frac{f^{\prime \prime}\left(r e^{i \theta}\right)}{f^{\prime}\left(r e^{i \theta}\right)}\right\} d \theta<\pi\left(\theta_{1}<\theta_{2}\right)
$$

which are the conditions Libera and Robertson [6] derived for closeto-convex functions in $0<|z|<1$.

THEOREM 3. Let $f(z) \in C \Sigma_{0}(p)$; then $f(z)$ is convex and univalent in $|z|<\rho_{2}$, where $\rho_{2}$ is the smallest positive root of 


$$
p\left(1+r^{4}\right)-\left(1+4 p^{2}\right)\left(r+r^{3}\right)-2\left(3+2 p+3 p^{2}\right) r^{2}=0 .
$$

Proof. For a function $f(z) \in C \Sigma_{0}(p)$ there exist functions $P(z) \in \mathcal{P}$ and $g(z) \in \Sigma(p)$ such that

$$
f^{\prime}(z)=g^{\prime}(z) P(z)
$$

for $|z|<1$. Thus we have

$$
\operatorname{Re}\left\{1+z \frac{f^{\prime \prime}(z)}{f^{\prime}(z)}\right\}=\operatorname{Re}\left\{1+z \frac{g^{\prime \prime}(z)}{g^{\prime}(z)}\right\}+\operatorname{Re}\left\{z \frac{P^{\prime}(z)}{P(z)}\right\} .
$$

Using (6) and a result of MacGregor [7], we have

$$
\begin{array}{r}
\operatorname{Re}\left\{1+z \frac{f^{\prime \prime}(z)}{f^{\prime}(z)}\right\} \geqq \frac{p-\left(1+3 p+p^{2}\right)\left(r+r^{2}\right)+p r^{3}}{(p+r)(1+p r)(1-r)}-\frac{2 r}{1-r^{2}} \\
=\frac{p\left(1+r^{4}\right)-\left(1+4 p+p^{2}\right)\left(r+r^{3}\right)-2\left(3+2 p+3 p^{2}\right) r^{2}}{\left(1-r^{2}\right)(p+r)(1+p r)}
\end{array}
$$

for $z=r e^{i \theta}$ and $r<p$. This completes the proof.

A corresponding theorem may be proved for the case where the function $g(z)$ in $(7)$ is meromorphic. If the function $g(z) \in K(p)$, then (8) may be replaced with

$$
\int_{\theta_{1}}^{\theta_{2}} \operatorname{Re}\left\{1+r e^{i \theta} \frac{f^{\prime \prime}\left(r e^{i \theta}\right)}{f^{\prime}\left(r e^{i \theta}\right)}\right\} d \theta<\pi\left(\theta_{1}<\theta_{2}\right)
$$

for $r>\rho$, where $\rho$ is such that if $|z|>\rho$, then

$$
\operatorname{Re}\left\{1+z g^{\prime \prime}(z) / g^{\prime}(z)\right\} \leqq 0 .
$$

4. Starlike functions. Since functions in $U(p)$ map the disk onto the exterior of a bounded domain, the concept of starlike with respect to the origin or the point at infinity cannot be used. However, we may use Robertson's concept of star center points [8]. Let $U^{*}\left(p, w_{0}\right)$ denote the set of functions defined by: $f(z) \in U^{*}\left(p, w_{0}\right)$ if and only if $f(z) \in U(p), f(z) \neq w_{0}$ for all $z \in E$, and there exists a $\rho, p<\rho<1$, such that

$$
\operatorname{Re}\left\{z f^{\prime}(z) /\left(f(z)-w_{0}\right)\right\} \leqq 0
$$

for $\rho<|z|<1$. Functions in $U^{*}\left(p, w_{0}\right)$ map $E$ onto the exterior of sets which are starlike with respect to $w_{0}$. 
TheOREM 4. Let $f(z) \in U^{*}\left(p, w_{0}\right)$ and $0<p<2-\sqrt{ } 3$. Then

$$
\operatorname{Re}\left\{z f^{\prime}(z) /\left(f(z)-w_{0}\right)\right\} \leqq 0
$$

for all $|z|>\rho=\left[1-6 p^{2}+p^{4}-\left(1-p^{2}\right)\left(p^{2}-14 p^{2}+1\right)^{1 / 2}\right] \cdot\left[2 p+2 p^{3}\right]^{-1}$.

Proof. If (9) holds, then there exists a function $P(z) \in \mathcal{P}$ such that

$$
z \frac{f^{\prime}(z)}{f(z)-w_{0}}+\frac{p}{z-p}-\frac{p z}{1-p z}=-P(z) .
$$

Taking real parts, we get

$$
\operatorname{Re}\left\{z \frac{f^{\prime}(z)}{f(z)-w_{0}}\right\}=p \operatorname{Re}\left\{\frac{z}{1-p z}-\frac{1}{z-p}\right\}-\operatorname{Re}\{P(z)\} .
$$

We now need the following lemma:

Lemma 4 [9]. Let $Q(r, \theta)$ be as defined in Lemma 1, and

$$
R(p)=\frac{1-6 p^{2}+p^{4}-\left(1-p^{2}\right)\left(p^{4}-14 p^{2}+1\right)^{1 / 2}}{2 p+2 p^{3}} .
$$

If $0<p<2-\sqrt{ } 3$ and $R(p)<r<1$, then

$$
\max _{0 \leqq \theta<2 \pi} \operatorname{Re}\{Q(r, \theta)\}=\operatorname{Re} Q(r, \pi)=\frac{\left(1-r^{2}\right)}{(r+p)(1+r p)} .
$$

Using Lemma 4, we have

$$
\begin{aligned}
\operatorname{Re}\left\{z \frac{f^{\prime}(z)}{f(z)-w_{0}}\right\} & \leqq \frac{p\left(1-r^{2}\right)}{(r+p)(1+p r)}-\frac{1-r}{1+r} \\
& =-\frac{(1-r)(1-p)^{1 / 2} r}{(1+r)(r+p)(1+p r)}<0
\end{aligned}
$$

for all $|z|=r>R(p)$.

\section{REFERENCES}

1. A. W. Goodman, Functions typically-real and meromorphic in the unit circle, Trans. Amer. Math. Soc. 81 (1956), 92-105. MR 17, 724.

2. J. A. Jenkins, On a conjecture of Goodman concerning meromorphic univalent functions, Michigan Math. J. 9 (1962), 25-27. MR 24 \#A2017.

3. W. Kaplan, Close-to-convex schlicht functions, Michigan Math. J. 1 (1952), 169-185. MR 14, 966.

4. Y. Komatu, Note on the theory of conformal representation by meromorphic functions. I, II, Proc. Japan Acad. 21 (1945), 269-284. MR 11, 170. 
5. K. Ladegast, Beiträge zur Theorie der schlichten Funktionen, Math. Z. 58 (1953), 115-159. MR 15, 24.

6. R. J. Libera and M. S. Robertson, Meromorphic close-to-convex functions, Michigan Math. J. 8 (1961), 165-175. MR 24 \#A2014.

7. T. H. MacGregor, The radius of univalence of certain analytic functions, Proc. Amer. Math. Soc. 14 (1963), 514-520. MR $26 \# 6388$.

8. M. S. Robertson, Star center points of multivalent functions, Duke Math. J. 12 (1945), 669-684. MR 7, 379.

9. W. C. Royster, Convex meromorphic functions, MacIntyre Memorial Volume, Ohio University, Athens, Ohio, 1970.

NaSA, Manned Spacecraft Center, Houston, Texas 77058 